\title{
HOW TO QUANTIFY THE VIBRATION REDUCTION OF RAILWAY TRACKS
}

\author{
L. Auersch \\ ${ }^{1}$ Federal Institute of Material Research and Testing \\ 12200 Berlin, Germany \\ e-mail: Lutz.auersch-saworski@bam.de
}

Keywords: Railway induced ground vibration, mitigation measures, railway tracks, finiteelement boundary-element method, wavenumber integrals, 1-D insertion loss.

\begin{abstract}
Railway tracks, which reduce the ground vibration, are necessary if the trains are passing near to building. Today the reduction effect of railway tracks is often determined by the complex (time domain) simulation of the passage of a complete train. This method of evaluating the reduction is very time consuming and the results include some arbitrary scatter. It is worth to look for simpler methods which yield clearer results in shorter time. This contribution presents the combined finite-element boundary-element method as a detailed method for the track-soil interaction and, on the other hand, some possible simplifications. One important idea for the reduction assessment is to split the reduction in a vehicle-track interaction part and a track transfer part. The track transfer is not influenced by the vehicle and by single axle loads and it can be calculated as a homogeneous problem along the track. The reduction of some tracks (for example of floating slab tracks) can be dominated by this track transfer function whereas other tracks (for example tracks with soft rail pads) are completely determined by the vehicle-track-interaction part. The different force transfer functions and the ground vibration ratios of tracks with elastic rail pads or elastic sleeper pads are shown as a result of the FEBEM model under a fixed harmonic axle load. The force transfer proves to be a useful quantity for to assess the vibration reduction of railway tracks.
\end{abstract}




\section{INTRODUCTION}

Trains are passing over tracks, and the irregularities of the wheel and the track yield dynamic loads on the track and the soil. Waves are generated and are propagating through the soil. The ground vibration excites buildings in the vicinity and can annoy persons who live or work inside them. Nuisance problems must be solved by countermeasures at the vehicle, the track or the transmission path. This contribution deals with special countermeasures at the track, elastic rail pads and sleeper pads.

The contribution describes different methods for the analysis of the track-soil system and, in the third section the method of vehicle-track interaction. Results are presented firstly for the track and vehicle-track system and, secondly, for the ground vibration.

\section{METHODS FOR THE ANALYSIS OF THE TRACK}

Four different methods will be presented which allow a dynamic analysis of the track. The most rigorous method is the combined 3-dimensional finite-element boundary-element method. The second method for a multi beam on a continuous soil can be applied for a continuous slab tracks. Whereas Fourier wavenumber integrals must be used for the continuous soil, the solution for a multi beam on a Winkler soil can be solved in space domain. Some simplifications are valid for the force transfer of the track which can be exploited by insertion loss formula.

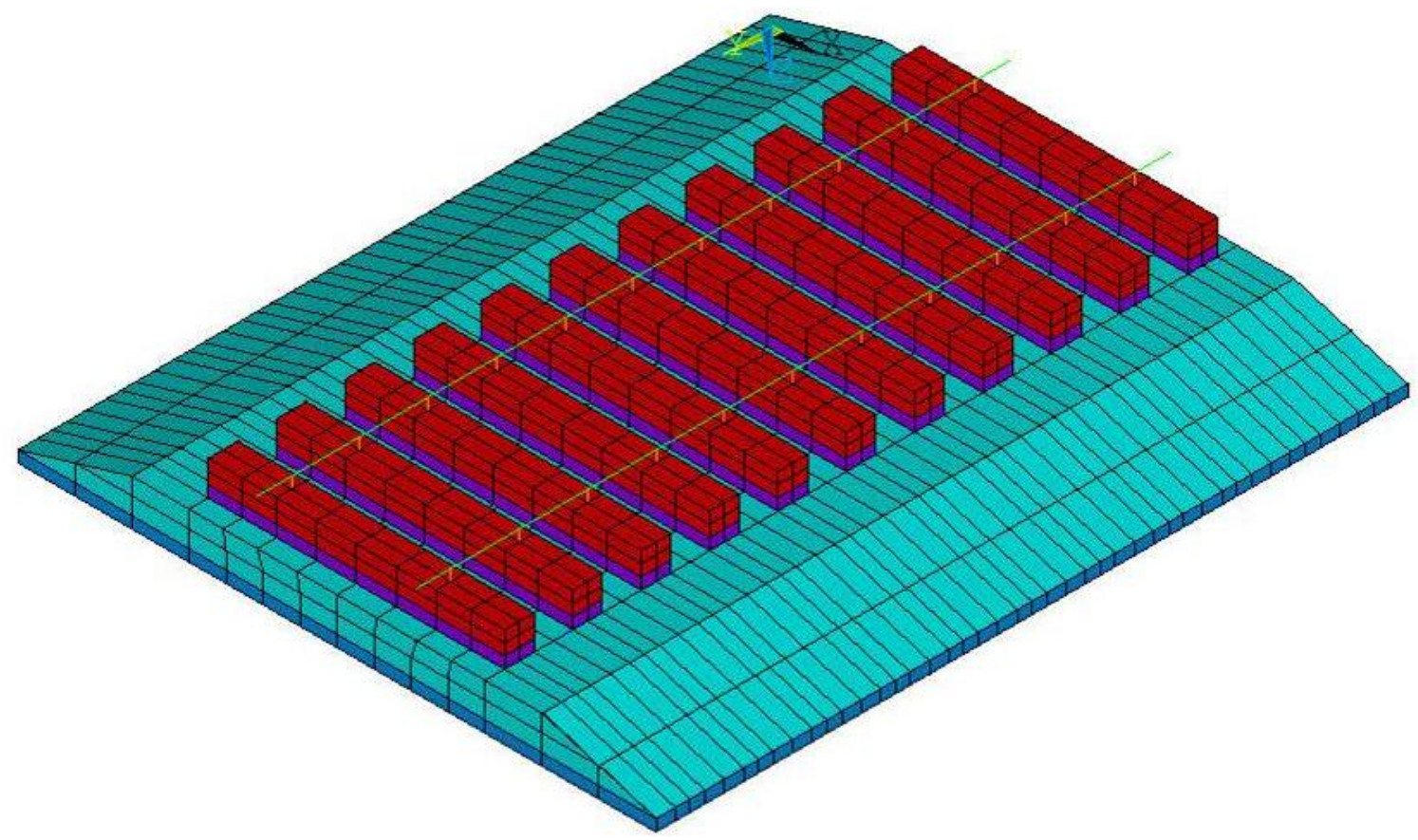

Figure 1: Finite element model of the ballasted track with elastic rail pads, sleeper pads and ballast mat.

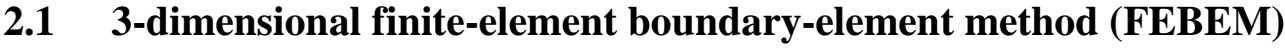

The track-soil systems are calculated most detailed by the combined finite-element boundary-element method [1]. The track including the rails, rail pads, sleepers, under sleeper pads, and ballast is modeled by the finite element method (Fig. 1) whereas the homogeneous or layered soil is modeled by the boundary element method. The dynamic stiffness matrix of the soil is established by using the Green's functions of an elastic layered half-space [2]. All calculations (Green's functions, boundary matrix and finite element matrices) are performed in frequency domain. Special additional methods (within the FEBEM) have been developed 
for infinite tracks [3] which are also applied to this study. The track displacement $u_{R}$ and the total force $F_{S}$ that is acting on the soil are evaluated from the results. As the Green's function of the homogeneous or layered soil are available, the ground vibration $v_{i}$ at distances $x_{i}$ away from the track can also be calculated.

wheelset mass

bending stiffness of the rails (UIC60)

mass per length of the rails

distance of the rail pads

stiffness of the rail pads

modulus of elasticity of the sleepers

mass density of the sleepers

length of the sleepers

height of the sleepers

width of the sleepers

stiffness of the sleeper pads

shear modulus of the ballast

shear wave velocity of the ballast

smaller width of the ballast

larger width of the ballast

height of the ballast

shear modulus of the soil

shear wave velocity of the soil

mass density of the soil and ballast

Poisson's ratio of the soil and ballast

hysteretic damping of the soil and ballast

hysteretic damping of the elastic elements

$$
\begin{aligned}
& m_{W}=1000, \underline{1500}, 2000,3000 \mathrm{~kg} \\
& E I=12.610^{6} \mathrm{Nm}^{2}, \\
& m_{R}=2 \times 60 \mathrm{~kg} / \mathrm{m}, \\
& d=0.6 \mathrm{~m}, \\
& k_{P}=5,10,20,40,80,150, \underline{300} 10^{6} \mathrm{~N} / \mathrm{m}, \\
& E_{S}=310^{10} \mathrm{~N} / \mathrm{m}^{2}, \\
& \rho_{S}=0.63,1.25, \underline{2.5}, 510^{3} \mathrm{~kg} / \mathrm{m}^{3}, \\
& a_{S}=2.6 \mathrm{~m}, \\
& h_{S}=0.2 \mathrm{~m}, \\
& b_{S}=\underline{0.26}, 0.52 \mathrm{~m}, \\
& k_{S}=12.5,25, \underline{50}, 100,20010^{6} \mathrm{~N} / \mathrm{m}, \\
& G_{B}=2,4.5,8, \underline{18} 10^{7} \mathrm{~N} / \mathrm{m}^{2}, \\
& v_{S B}=100,150,200, \underline{300} \mathrm{~m} / \mathrm{s} \\
& a_{B 1}=3.6 \mathrm{~m}, \\
& a_{B 2}=5.6 \mathrm{~m}, \\
& h_{B}=0.3 \mathrm{~m}, \\
& G=2,4.5, \underline{8}, 18,5010^{7} \mathrm{~N} / \mathrm{m}^{2}, \\
& v_{S}=100,150, \underline{200}, 300,500 \mathrm{~m} / \mathrm{s}, \\
& \rho=210^{3} \mathrm{~kg} / \mathrm{m}^{3}, \\
& v=0.33, \\
& D=2.5 \%, \\
& D_{P}=D_{S}=10 \% .
\end{aligned}
$$

Table 1: Parameters of the vehicle-track-soil systems (standard parameters are underlined).

\subsection{Multi beam on continuous soil}

The multi beam on continuous soil is solved in frequency-wavenumber domain [4]. Each beam is described by the bending stiffness $E I_{j}$ and the mass per length $m_{j}{ }^{\prime}$ which are assembled in a diagonal stiffness matrix EI and a diagonal mass matrix m'. The global stiffness matrix $\mathbf{K}^{\prime}$ is a $n \times n$ matrix assembled from the $2 \times 2$ dynamic stiffness matrices of each support section. The multi-beam system fulfils the set of differential equations for the beam displacements $\mathbf{u}$ under the track load $\mathbf{F}_{\mathbf{T}}$,

$$
\text { Elu' '"'+m' } \ddot{\mathbf{u}}+\mathbf{K}^{\prime} \mathbf{u}=\mathbf{F}_{\mathbf{T}}{ }^{\prime}
$$

The contact force $F{ }_{S}$ between the lowest beam and the soil is distributed uniformly across the width. The average displacement of the soil across the width is equal to the displacement of the lowest beam.

The dynamic stiffness of the multi-beam track model in the frequency-wavenumber domain reads as

$$
\mathbf{K}_{\mathbf{T}}=k_{y}{ }^{4} \mathbf{E I}-\omega^{2} \mathbf{m}^{\prime}+\mathbf{K}^{\prime}
$$

where $k_{y}$ is the wavenumber along the track axis. In order to couple the track and the soil, the dynamic soil stiffness $\mathbf{K}_{\mathbf{S}}$ for harmonic waves along the track [4] is added to the track stiffness 
(2) at the last diagonal element and the dynamic stiffness matrix of the track-soil system is established

$$
\mathbf{K}_{\mathrm{TS}}=\mathbf{K}_{\mathrm{T}}+\mathbf{K}_{\mathrm{S}}
$$

The displacements in the frequency-wavenumber domain are calculated by the inversion of this matrix

$$
\mathbf{u}=\mathbf{K}_{\mathrm{TS}}^{-1} \mathbf{F}_{\mathbf{T}},
$$

The wavenumber transform of the vertical point load $\mathbf{F}_{\mathbf{T}}{ }^{\prime}(y)=\mathbf{F}_{\mathbf{T}} \delta(y)$ is the constant force $\mathbf{F}_{\mathbf{T}}$. The displacements along the track can be calculated by the inverse Fourier transformation as

$$
\mathbf{u}(y, \omega)=\frac{1}{2 \pi} \int_{-\infty}^{+\infty} \mathbf{K}_{T S}^{-1}\left(k_{y}, \omega\right) \mathbf{F}_{\mathbf{T}} e^{i k_{y} y} d k_{y}
$$

The force distribution on the track-soil interface can then be calculated by a similar Fourier integral

$$
F^{\prime}{ }_{S}(y, \omega)=\frac{1}{2 \pi} \int_{-\infty}^{+\infty} K_{S}\left(k_{y}, \omega\right) u_{S}\left(k_{y}, \omega\right) e^{i k_{y} y} d k_{y}
$$

using the displacements of the track-soil interface $u_{S}\left(k_{y}, \omega\right)$ and the soil stiffness $K_{S}\left(k_{y}, \omega\right)$.

Finally, the total force that acts on the track-soil interface is calculated as the integral over the infinite track length

$$
F_{S}(\omega)=\int_{-\infty}^{+\infty} F^{\prime}{ }_{S}(y, \omega) d y=F^{\prime}{ }_{S}\left(k_{y}=0, \omega\right)=K_{S}\left(k_{y}=0, \omega\right) u_{S}\left(k_{y}=0, \omega\right)
$$

This soil force can easily be obtained as the transformed integrand at $k_{y}=0$ without any integration. Moreover, the total force computation corresponds to a two-dimensional analysis across the track.

The ground vibration, however, needs the most laborious calculation. A double Fourier transform along $k_{x}$ and $k_{y}$ must be performed

$$
u(x, y, \omega)=\frac{1}{(2 \pi)^{2}} \int_{-\infty}^{+\infty} \int_{-\infty}^{+\infty} H\left(k_{x}, k_{y}, \omega\right) K_{S}\left(k_{y}, \omega\right) u_{S}\left(k_{y}, \omega\right) p_{1}\left(k_{x}\right) e^{i\left(k_{x} x+k_{y} y\right)} d k_{x} d k_{y}
$$

to obtain the soil displacements at any position away from the track.

\subsection{Multi beam on Winkler soil}

In general, the Winkler soil is not an adequate model of the real soil [1], but for certain conditions a rough approximation has been achieved [5]. In case of a Winkler soil, the soil stiffness does not depend on the wavenumber and can be expressed as a spring and a damper force so that the solution can be obtained in space domain.

The displacement solution $\mathbf{u}$ for this track system is found as

$$
\mathbf{u}(y)=\sum_{j=1}^{2 n} A_{j} \mathbf{v}_{\mathrm{j}} e^{k_{j} y}
$$

where the $k_{i}$ are the solution of the eigenvalue problem 


$$
\left(\mathbf{E I} k^{4}-\mathbf{m}^{\prime} \omega^{2}+\mathbf{K}^{\prime}\right) \mathbf{u}=\mathbf{0}
$$

and $\mathbf{v}_{\mathrm{j}}$ the corresponding eigenvectors, see [6] for more details. In case of a single beam on Winkler soil, the compliance solution can be expressed as

$$
\frac{u_{R}}{F_{T}}=\frac{1}{2 \sqrt{2} E I^{0.25}\left(k^{\prime}-m^{\prime} \omega^{2}\right)^{0.75}} .
$$

For the force transfer $F_{S} / F_{T}$ of the track, the following general relation holds

$$
F_{S}=\int_{-\infty}^{\infty} F_{S}{ }^{\prime} d y=\int_{-\infty}^{\infty} \frac{F_{S}{ }^{\prime}}{F_{T}{ }^{\prime}} F_{T}{ }^{\prime} d y=\frac{F_{S}{ }^{\prime}}{F_{T}{ }^{\prime}} \int_{-\infty}^{\infty} F_{T}{ }^{\prime} d y=\frac{F_{S}{ }^{\prime}}{F_{T}{ }^{\prime}} F_{T}
$$

and that means

$$
\frac{F_{S}}{F_{T}}=\frac{F_{S}{ }^{\prime}}{F_{T}{ }^{\prime}}
$$

that the ratio between the soil force $F_{S}$ (the sum of all soil forces distributed along the track) and the force $F_{T}$ that is acting on the track (the dynamic axle load) is the same as the ratio $F_{S}{ }^{\prime} / F_{T}$ ' for each support element independent of the load distribution by the bending stiffness of the beams.

\subsection{1-dimensional insertion loss formula for the force transfer of the track}

As the force transfer of the track can be analysed by the 1-dimensional support chains, the insertion loss formula

$$
\frac{F_{S, \text { without }}}{F_{S, \text { with }}}=\frac{\frac{1}{K_{Q}}+\frac{1}{K_{R}}+\frac{1}{k_{I}}}{\frac{1}{K_{Q}}+\frac{1}{K_{R}}}=1+\frac{1}{k_{I}} \frac{K_{Q} K_{R}}{K_{Q}+K_{R}}
$$

can be applied to get the effect of the insertion of an elastic element with a spring stiffness $k_{I}$, where $K_{Q}$ and $K_{R}$ are the dynamic stiffnesses of the source and the receiver, see the Appendix for the derivation.

This formula is valid for the force transfer of the track. To apply the formula also to the whole vehicle-track system as in [7] and [8], a track- and frequency-dependent track length must be used to get the same results as for the beam on Winkler soil [6]. The 2-dimensional beam-on-Winkler-soil model is almost as simple as the 1-dimensional model and the determination of realistic parameters is more straightforward. Generally, the simple 1dimensional and 2-dimenional results must be checked by more detailed methods for the track and the soil.

\section{METHODS FOR THE VEHICLE-TRACK INTERACTION}

The influence of the vehicle on the track and ground vibration can be calculated in a second step. The compliance of the track is inverted to the track stiffness $K_{T}=\left(u_{R} / F_{T}\right)^{-1}$. A corresponding dynamic stiffness $K_{V}$ of the vehicle can be calculated by multi-body dynamics [9]. It can be approximated by the inertia of the wheelset $K_{V}=-m_{W} \omega^{2}$ in the frequency range of interest. The vehicle is excited by the irregularities $s$ of the track (alignment and rail roughness) and the vehicle (out-of-roundness and roughness of the wheel). 
The transfer function between the irregularities $s$ and the force $F_{T}$ on the track can be calulated from these two dynamic stiffnesses as $[1,3]$

$$
\frac{F_{T}}{s}=-\frac{K_{T} K_{V}}{K_{T}+K_{V}}
$$

The vehicle-track interaction can also be expressed as

$$
\frac{F_{T}}{F_{V}}=\frac{K_{T}}{K_{T}+K_{V}}
$$

if the equivalent excitation force $F_{V}=-K_{V} S$ is used. This is a second force transfer function which has to be multiplied with the force transfer function $F_{S} / F_{T}$ of the track to get the total force transfer function

$$
\frac{F_{S}}{F_{V}}=\frac{F_{S}}{F_{T}} \frac{F_{T}}{F_{V}}
$$

\section{FORCE TRANSFER OF THE TRACK AND VEHICLE-TRACK SYSTEM}

Two types of elastic elements are considered, elastic rail pads between rail and sleeper and elastic sleeper pads between sleeper and ballast. The stiffness of these elements is varied in a wide range, the rail pad stiffness as $k_{P}=5,10,20,40,80,15010^{6} \mathrm{~N} / \mathrm{m}$ and the sleeper pad stiffness as $k_{S}=12.5,25,50,100,20010^{6} \mathrm{~N} / \mathrm{m}$. By this choice, the stiffness per sleeper is approximately the same for rail and sleeper pads. As a reference, a ballasted track without sleeper pad and with a stiff rail pad of $k_{P}=30010^{6} \mathrm{~N} / \mathrm{m}$ is used.
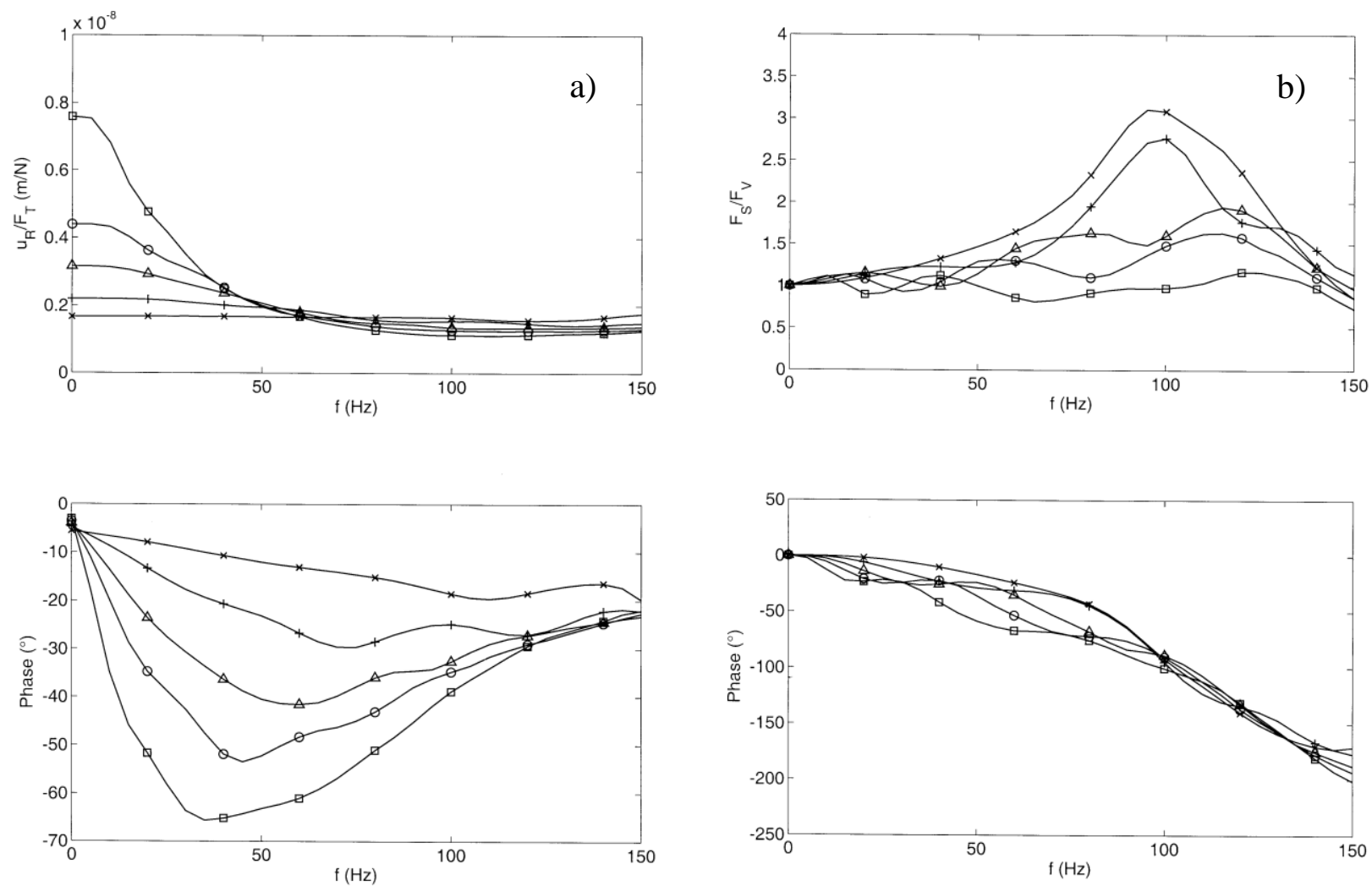

Figure 2: Compliance (a) and total force transfer (b) (amplitude and phase) of a ballasted track on different soils, $v_{S}=\square 100, \bigcirc 150, \triangle 200,+300, \times 500 \mathrm{~m} / \mathrm{s}$ 

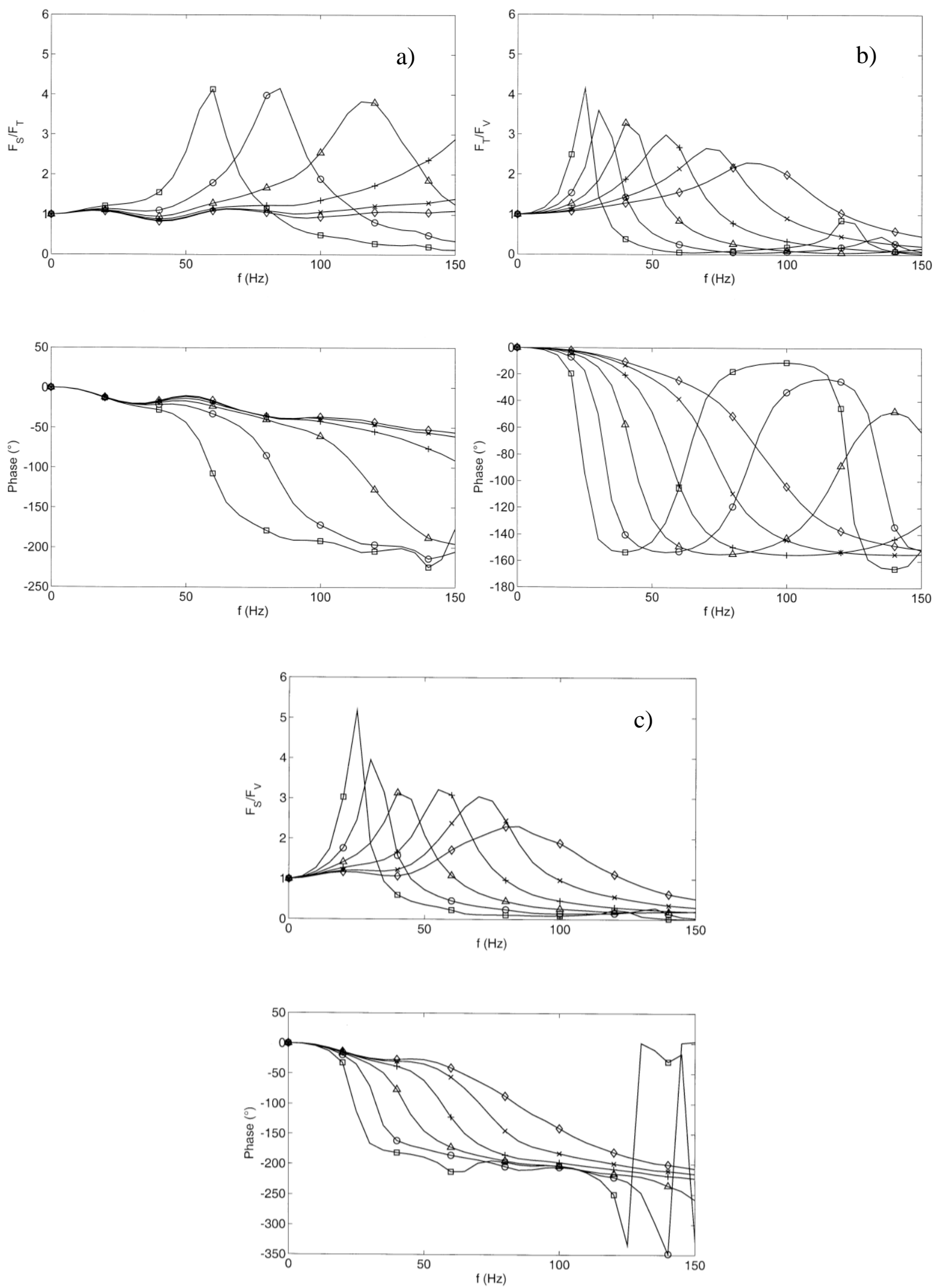

Figure 3: Tracks on different elastic rail pads $k_{P}=\square 5, \bigcirc 10, \triangle 20,+40, \times 80, \diamond 150 \mathrm{kN} / \mathrm{mm}$, force transfer of the track (a), of the vehicle-track interaction (b) and the total force transfer (c) as amplitude and phase. 

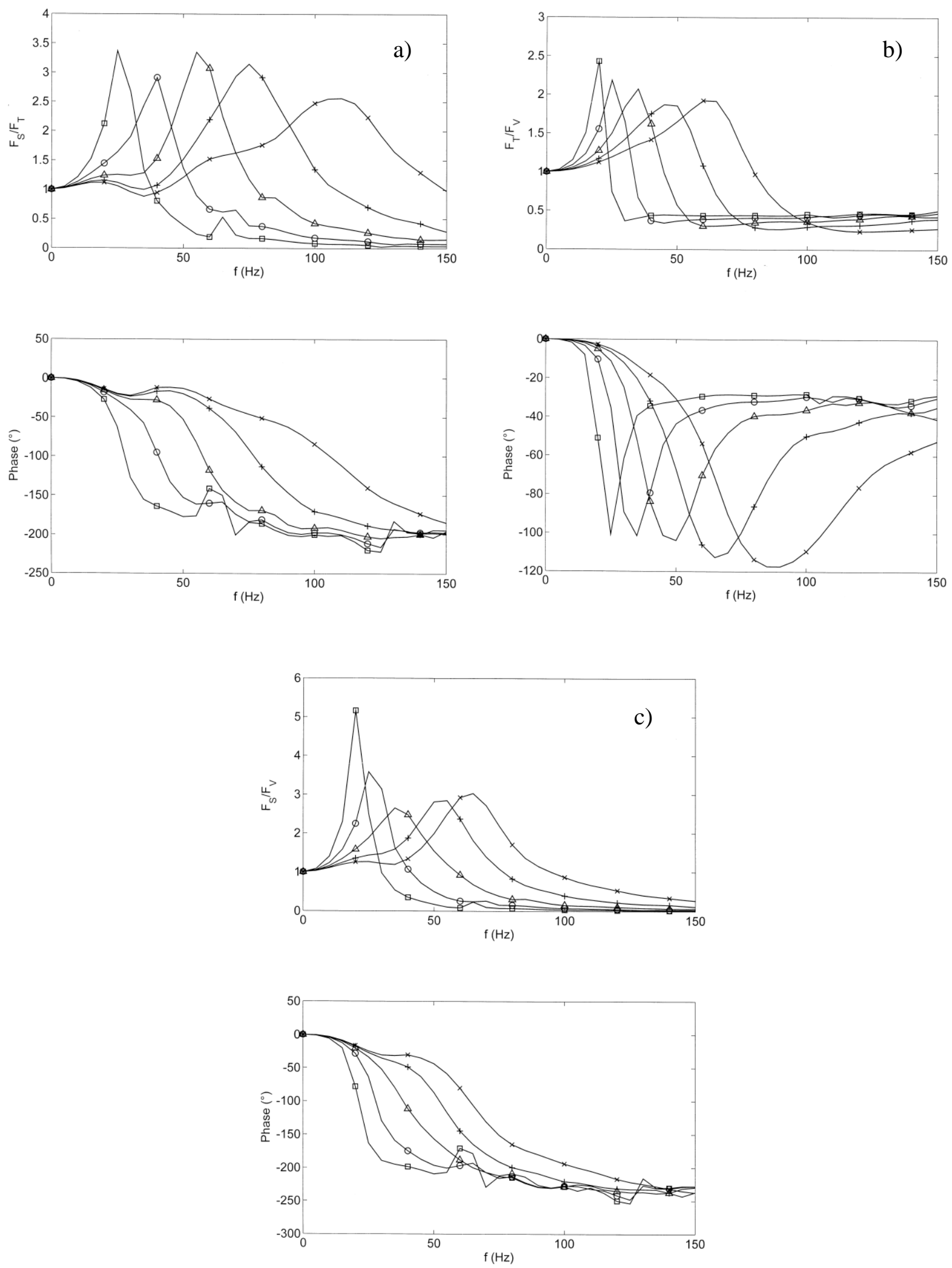

Figure 4: Tracks on different elastic sleeper pads $k_{S}=\square 12.5, \bigcirc 25, \triangle 50,+100, \times 200 \mathrm{kN} / \mathrm{mm}$, force transfer of the track (a), of the vehicle-track interaction (b) and the total force transfer (c) as amplitude and phase. 
The reference ballasted track is analysed for the track compliance $u_{R} / F_{T}$ (Fig. 2a) and the total force transfer $F_{S} / F_{V}$ (Fig. 2b). All results are presented as frequency dependent amplitude and phase. The influence of different soils characterized by their shear wave velocities $v_{S}$ is demonstrated. A strong influence of the soil on the low-frequency compliance can be observed. At higher frequencies, the compliances are almost the same for the different soils. Therefore, similar resonance frequencies of the vehicle-track-soil systems are found at about 100-120 Hz. In general, the force transfer of a track without any elastic element is close to the value 1 , and the resonance amplifications are weak due to the radiation damping into the infinite soil, except for the two stiffest soils.

The track with elastic rail pads is analysed for the three different force transfers of the track (Fig. 3a), the vehicle-track interaction (Fig. 3b), and the total force transfer (Fig. 3c). The resonance frequencies of a track with elastic rail pads are relatively high due to the small rail mass. The resonances are observed for the three softest rail pads at 60 to $150 \mathrm{~Hz}$, whereas no track resonance can be found below $150 \mathrm{~Hz}$ for normal pad stiffnesses. The reduction effect of the rail pads is based on the greater wheelset mass and the vehicle-track interaction. Compared to the track system without wheelset mass (Fig. 3a), the complete vehicle-track system yields considerably lower resonance frequencies (Fig. 3b,c). The resonance frequencies for the whole vehicle-track system are in the range of 25 to $90 \mathrm{~Hz}$ for the different rail pads which means an increase according to $f_{0} \sim k_{P}{ }^{0.35}$. For frequencies higher than the resonance frequency, a clear reduction can be found. The reduction due to the elastic rail pads is $F_{S} / F_{V}=0.1$ to 1.8 at $100 \mathrm{~Hz}$. That means that the two stiffest rail pads do not yield a reduction at $100 \mathrm{~Hz}$, only at $150 \mathrm{~Hz}$ with values of 0.5 and 0.3 . The transfer function $F_{T} / F_{V}$ of the vehicle-track interaction and the total force transfer $F_{S} / F_{V}$ are close together in case of normal rail pads. The reduction effects of the rail pads are dominated by the vehicle-track interaction. For other track systems such as floating slab track it is quite opposite. The reduction effect of floating slab tracks is completely determined by the force transfer of the track [4].

The track with elastic sleeper pads is analysed in Figures 4a,b,c for the same three force transfer functions as the track with rail pads. The force transfer of the track shows the resonance frequencies of the track (Fig. 4a). All under sleeper pads yield track-pad resonances below $150 \mathrm{~Hz}$, starting at $25 \mathrm{~Hz}$ for the softest and ending at $110 \mathrm{~Hz}$ for the stiffest sleeper pad. Including the wheelset mass yields a somewhat lower vehicle-track resonance between 20 and $60 \mathrm{~Hz}$ (Fig. 4b). The vehicle-track transfer function $F_{T} / F_{V}$ drops down at the vehicletrack resonance frequencies and keeps an almost constant value of $F_{T} / F_{V}=0.35$ for higher frequencies. The total force transfer function, however, has a continuous decrease after the resonance which is determined by the track transfer function $F_{S} / F_{T}$. The vehicle-track interaction yields the reduction just after the vehicle-track resonance and contributes a constant reduction at high frequencies. The total force transfer owing to the elastic sleeper pads can be quantified as $F_{S} / F_{V}=0.016$ to 0.9 at $100 \mathrm{~Hz}$ and $F_{S} / F_{V}=0.07$ to 0.27 at $150 \mathrm{~Hz}$ where the softest pads yield the strongest reduction. Below $100 \mathrm{~Hz}$, only softer under sleeper pads yield considerable reductions. Around the vehicle-track resonance, all sleeper and rail pads yield resonance amplifications of $F_{S} / F_{V}=2$ to 5, the highest amplifications for the softest pads. The force transfer functions for the different sleeper pads are also presented as the results of the beam-on-Winkler-soil model in Fig. 5 which are quite similar as in Fig. 4.

The influence of the mass of the sleeper is studied in Figures 6a,b,c: The sleeper-on-pad resonance is shifted from $80 \mathrm{~Hz}$, via 60 and $50 \mathrm{~Hz}$ to less than $40 \mathrm{~Hz}$ if the mass is doubled three times. The strong reduction above this resonance frequency can clearly be seen in Figure $6 a$ where the lowest amplitudes are found for the lowest resonance frequency. If the vehicletrack interaction is considered (Fig. 6b), the vehicle-track resonance frequencies are lower and 

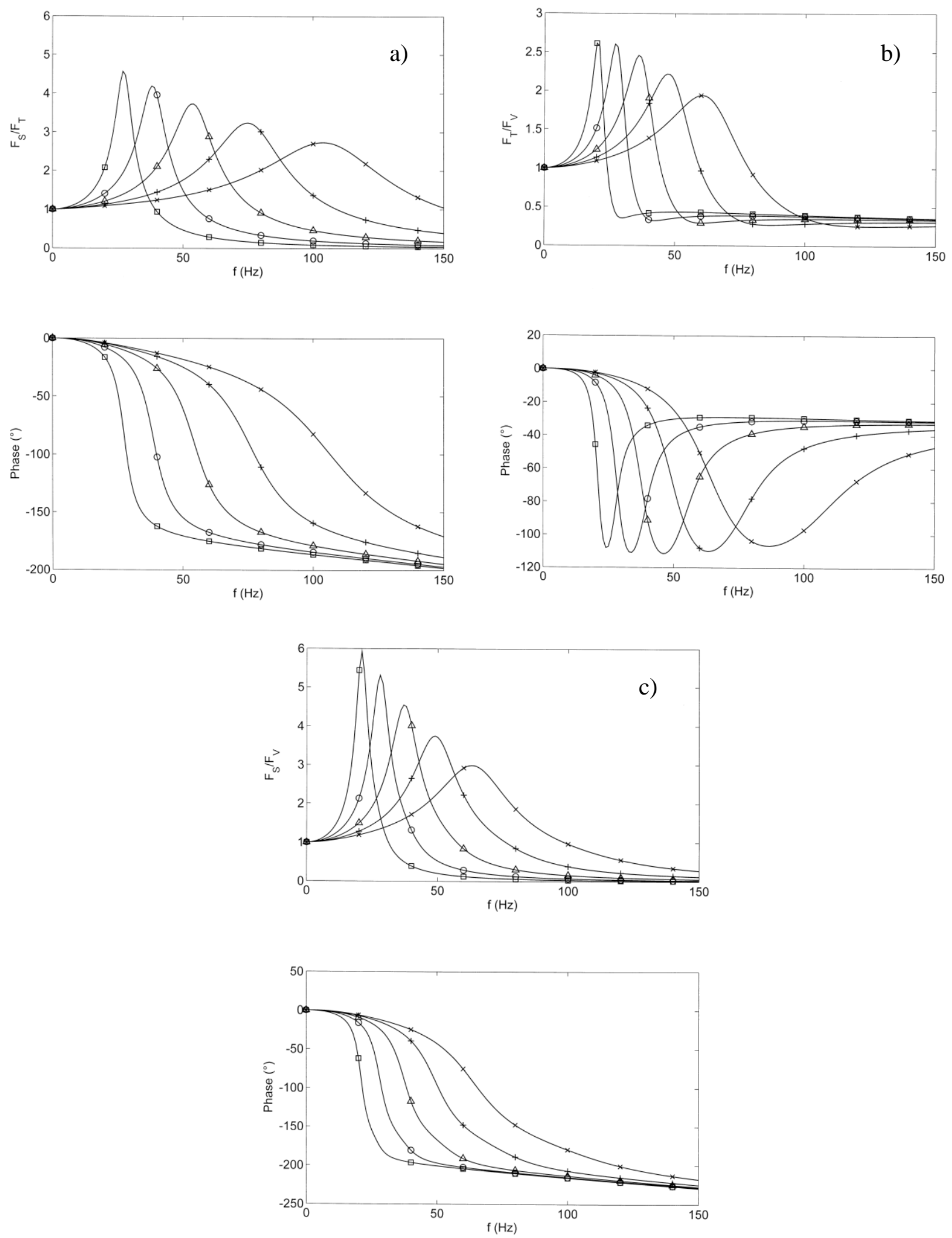

Figure 5: Tracks on different elastic sleeper pads $k_{S}=\square 12.5, \bigcirc 25, \triangle 50,+100, \times 200 \mathrm{kN} / \mathrm{mm}$, force transfer of the track (a), of the vehicle-track interaction (b) and the total force transfer (c) calculated for the beam-onWinkler-soil model. 

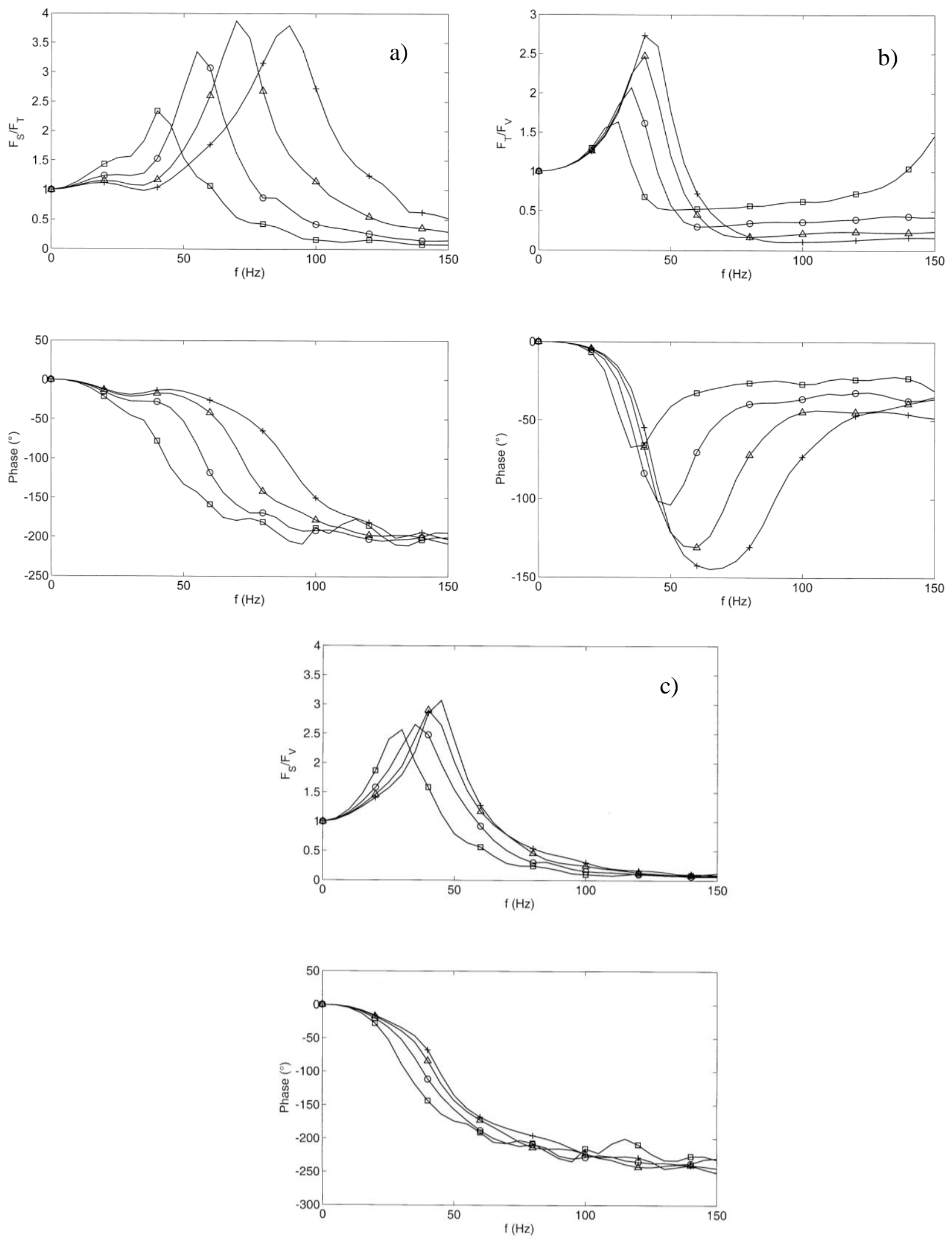

Figure 6: Track on elastic sleeper pads $k_{S}=50 \mathrm{kN} / \mathrm{mm}$, different sleeper masses $m_{S}=\square 1 / 4 m_{S 0}, \bigcirc 1 / 2 m_{S 0}$, $\triangle m_{S O},+2 m_{S 0}$, force transfer of the track (a), of the vehicle-track interaction (b) and the total force transfer (c) as amplitude and phase. 

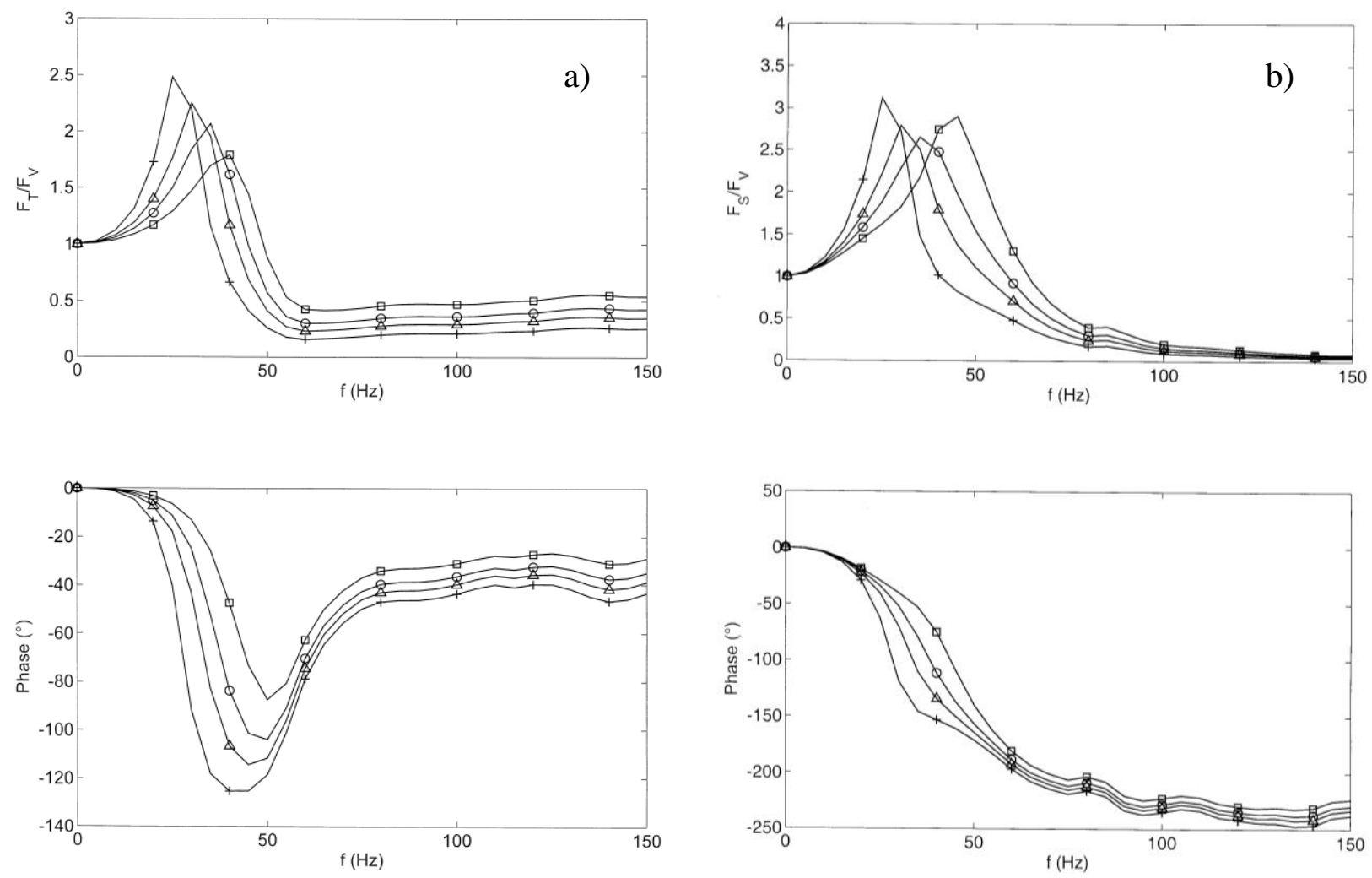

Figure 7: Track on elastic sleeper pads $k_{S}=50 \mathrm{kN} / \mathrm{mm}$, force transfer of the vehicle-track interaction (a) and the total force transfer (b) for different wheelset masses $m_{W}=\square 1000, \bigcirc 1500, \triangle 2000,+3000 \mathrm{~kg}$.
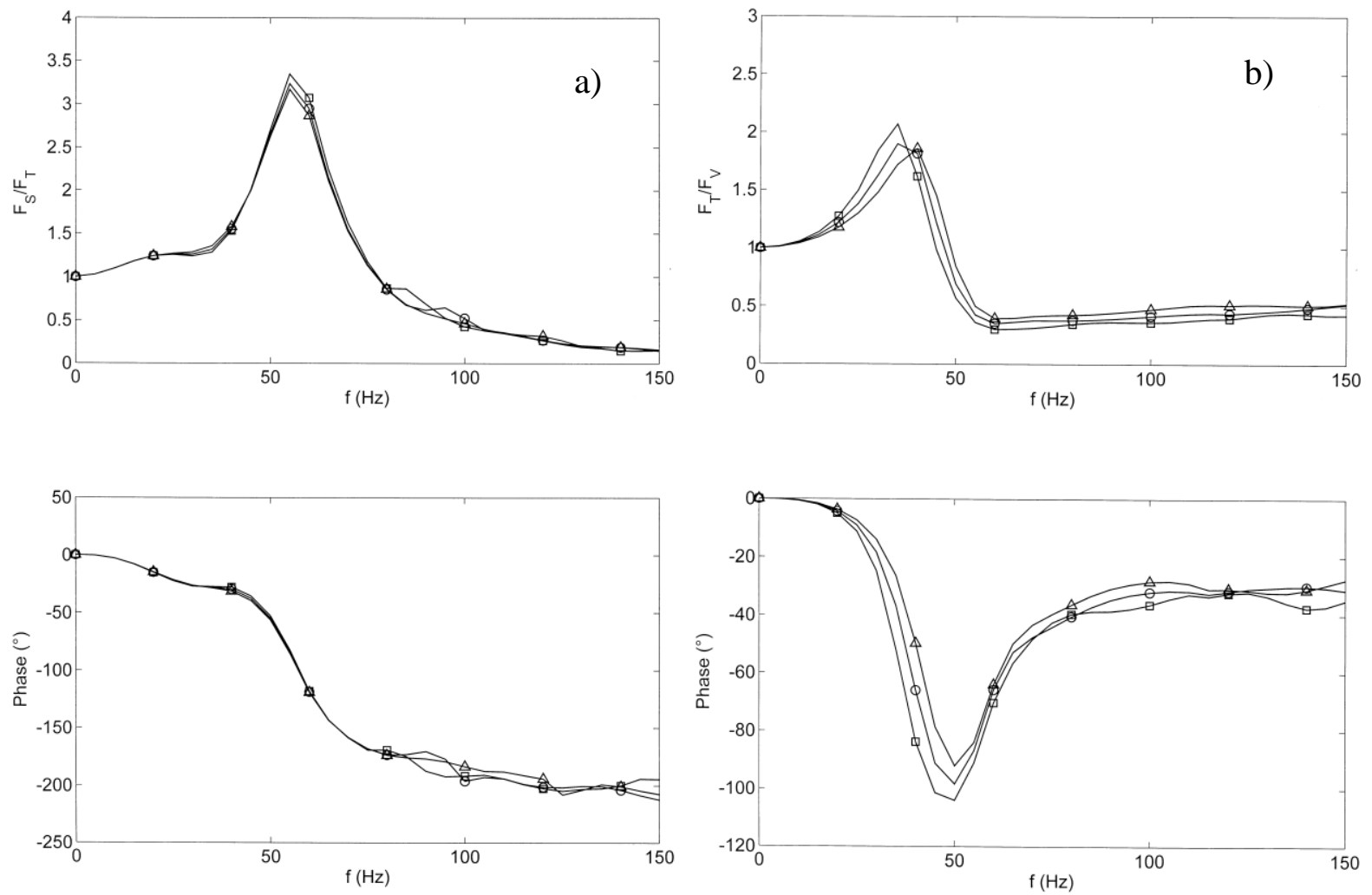

Figure 8: Tracks on elastic sleeper pads $k_{S}=50 \mathrm{kN} / \mathrm{mm}$ with different bending stiffnesses of the rails, $E I=\square E I_{0}$, $\bigcirc 2 E I_{0}, \triangle 4 E I_{0}$, force transfer of the track (a), of the vehicle-track interaction (b) and the total force transfer (c) as amplitude and phase. 
closer together. That means that the influence of the track mass on the whole system is weaker. The vehicle-track force transfer functions are almost constant at high frequencies, but with different reduction values which are lower for lower masses. The total transfer function in Figure $6 \mathrm{c}$ shows the vehicle-track resonances close together and also the high-frequency amplitudes close together what is due to the opposite effects of the track transfer and the vehicle-track transfer. Whereas the force reduction is similar for the small sleeper masses, a better reduction can be found for the double sleeper mass compared to the reference track for most frequencies above $32 \mathrm{~Hz}$.

The influence of the wheelset mass is presented in Figures $7 \mathrm{a}$ and $\mathrm{b}$. For higher wheelset masses the force reduction of the elastic sleeper pads can be improved. The force transfer of the track remains unchanged when the wheelset mass is varied. Therefore the additional effect is caused by the vehicle-track force transfer (Fig. 7b). The vehicle-track resonance frequency is shifted down to $25 \mathrm{~Hz}$ for a heavy wheelset of $m_{W}=3000 \mathrm{~kg}$. The transfer function drops down and keeps constant above the track resonance at $55 \mathrm{~Hz}$. The additional reduction depends on the mass of the wheelset and is in the range of $F_{T} / F_{V}=0.2$ to 0.55 for $m_{W}=3000$ to $1000 \mathrm{~kg}$. The continuous decrease of the total force transfer (Fig. 7b) at higher frequencies is due to the force transfer of the track with sleeper pads.

The minor influence of the bending stiffness of the track is demonstrated on Figure 8. As stated in the methods sections, the bending stiffness has no influence on the force transfer of the track (Fig. 8a). The influence on the track stiffness and the vehicle-track interaction (Fig. $8 b$ ) is weak. Although the bending stiffness is increased by a factor of 4 , the changes of the force transfer are less than 20 per cent.

Due to the higher track mass, the track with sleeper pads has lower resonance frequencies and stronger high-frequency reductions compared to the track with elastic rail pads.

\section{GROUND VIBRATION AND GROUND VIBRATION RATIOS}

So far, only the track behaviour has been considered. Now, the ground vibrations which are propagating away from the track are examined. The velocity amplitudes of the soil are calculated at the distances $x=5,10,15,20,25,30 \mathrm{~m}$ from the exciting force and are shown in Figures $9 \mathrm{a}, \mathrm{b}$ as admittance spectra $v / F(f)$ for the standard track and the track with sleeper pads of $k_{S}=50 \mathrm{kN} / \mathrm{mm}$. All admittance functions start with similar increasing amplitudes at low frequencies. The curves of the point load would continue to increase for almost all frequencies. Only the high-frequency far-field amplitudes are reduced due to the material damping. The standard track load yields almost constant ground vibration amplitudes at frequencies higher than $40 \mathrm{~Hz}$ what is due to the distribution of the load across the track width (see Auersch, $2005 \mathrm{~b}$ for details). The distribution of the force leads also to a marked minimum of amplitudes at 63 and $80 \mathrm{~Hz}$ if one wavelength of the soil fits into the sleeper length. The isolated track (Fig. 9b) has decreasing ground vibration amplitudes (admittance functions) at higher frequencies due to the additional reduction effect of the resiliently supported track mass.

To determine the effectiveness of the mitigation measure, the soil response (including the vehicle track interaction) of the isolated track is compared with the soil response of the unisolated track. The amplitude ratios between the isolated and un-isolated slab tracks of different sleeper pads are shown in Figures 9f,g for a near-field $(x=5 \mathrm{~m})$ and a far-field point $(x=30 \mathrm{~m})$. The amplitude ratios of the ground vibrations typically present the resonance of the vehicle and track on the sleeper pads and the reduction at higher frequencies. The ground vibration ratios under the track (Fig. 9e) are somewhat smaller than those of the near and far field. The vibration ratios for the track start with values smaller than 1 , and the resonance amplifications are also smaller. 

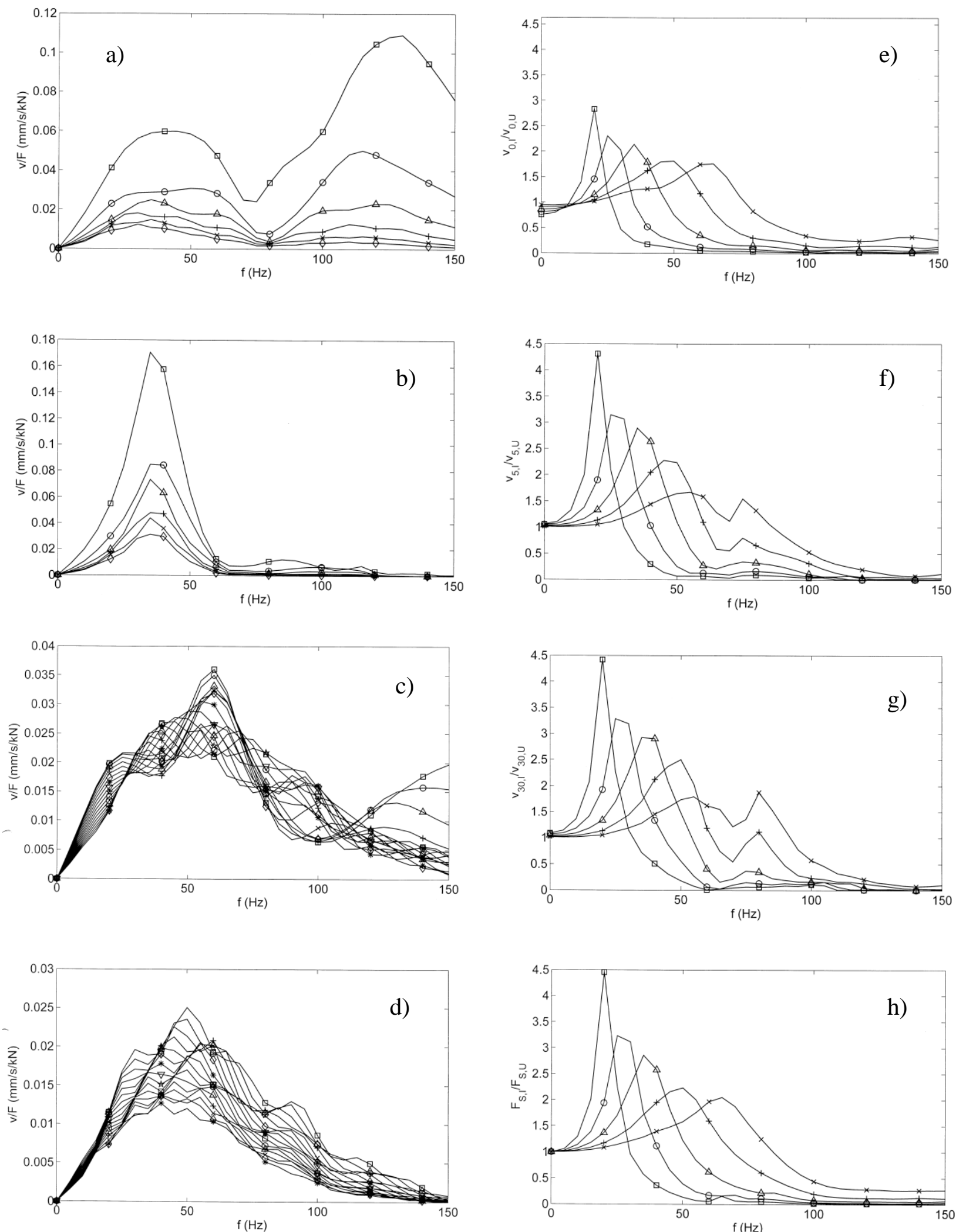

Figure 9: a, b) Ground vibration amplitudes at $\mathrm{x}=\square 5, \bigcirc 10, \triangle 15,+20, \times 25, \diamond 30 \mathrm{~m}$ distance from the load on the reference ballasted track (a) and the track with elastic sleeper pad of $k_{S}=50 \mathrm{kN} / \mathrm{mm}$. c, d) Ground vibration at $\mathrm{x}=8 \mathrm{~m}$ from the reference ballasted track, variation of the longitudinal distance from the load $y=0$ to $15 \mathrm{~m}$ (c) and $y=16$ to $31 \mathrm{~m}$. e, $\mathrm{f}, \mathrm{g}, \mathrm{h}$ ) ratios between isolated and un-isolated tracks, rail pads of $k_{S}=\square 12.5$,

25, $\triangle 50,+100, \times 200$, ground vibration at $x=0 \mathrm{~m}(\mathrm{e}), 5 \mathrm{~m}(\mathrm{f})$ and $30 \mathrm{~m}(\mathrm{~g})$, total soil force (h). 
These are effects of the load distribution along the track. The effects of the load distribution across the track yield some irregularities of the ground vibration ratios at 60 to $80 \mathrm{~Hz}$.

In Figure 9h, also the ratio of the soil forces for isolated and un-isolated tracks are presented. These force ratios are in good agreement with the ground vibration ratios. As the force ratios are very similar to the total force transfer $F_{S} / F_{V}$ in Figure $4 \mathrm{c}$, both force functions (force ratios and force transfer function) are good quantities to assess the mitigation potential of elastic elements of the track such as rail and sleeper pads. The force functions are smoother than the ground vibrations which depend on the specific load distribution under the track. Another method to get smoother results is to average the ground responses for different load positions which have less frequency-dependent variations for longitudinal distances $y>4 \mathrm{~m}$ as shown in Figures 9c,d.

\section{CONCLUSION}

Methods to assess the reduction of railway tracks have been presented. Detailed 3dimenional track-soil models are calculated by the combined finite-element boundary-element method (FEBEM). The force transfer is established as two parts, the force transfer of the track and the force transfer of the vehicle-track system. Simplifications are possible for the force transfer of the track where 2-dimensional beam-on-support models or 1-dimensional insertion loss formula are applicable if the track parameters are calibrated by detailed models.

Results have been shown for a track with rail pads, a track with sleeper pads and a track without any elastic element. Track resonances, vehicle-track resonances, and reduction effects at higher frequencies are found for the tracks with elastic elements. The best reduction values at $100 \mathrm{~Hz}$ for elastic rail and sleeper pads are 0.1 and 0.016 . These strong effects can only be achieved with very soft elements which, on the other hand, yield undesirable great displacements under the static train load. With constrained static displacements, the reductions of 0.27 and 0.18 at $100 \mathrm{~Hz}$ are more realistic. The mitigation effect is better for the sleeper pads owing to the higher track mass supported on the elastic element. Some parameter variations (pad stiffness, sleeper mass, wheelset mass, bending stiffness) are presented for the sleeper pads showing the vanishing influence of the bending stiffness on the track transfer and the possible improvement by heavier sleepers.

It has been found that the force reduction of the rail pads is almost completely determined by the vehicle-track interaction. The vehicle-track interaction has a considerable influence on the reduction of the sleeper pads, but the total reduction is also influenced by the force reduction of the track, which can be improved by heavier sleepers. For other track systems as ballasted tracks on ballast mats or floating slab tracks, the reduction effect is almost completely determined by the force reduction of the track.

The total soil force ratio is very close to the ground vibration ratio. Therefore, it may be concluded that the total force is the track quantity which is best suited to predict the reduction of the ground vibration by elastic track elements. 


\section{APPENDIX:}

EFFECTIVENESS OR INSERTION LOSS OF A MITIGATION MEASURE

The system is divided into a part close to the source (index Q) and a part close to the receiver (index R). The following dynamic stiffness relations hold for the two divided parts of the system

$$
\begin{aligned}
& K_{Q} u_{1}=-F_{1}+F \\
& K_{R} u_{2}=-F_{2}
\end{aligned}
$$

where an exciting force $\mathrm{F}$ has been introduced at the source side.

These formula yield directly the transfer function of the force for the case without any mitigation measure $\left(u_{1}=u_{2}, F_{1}=-F_{2}\right)$

$$
\left.\frac{F_{2}}{F}\right|_{\text {withhout }}=\frac{K_{R}}{K_{Q}+K_{R}}
$$

which means that the force transfer $F_{2} / F$ is the ratio of the dynamic stiffness of the receiver to the dynamic stiffness of the parallel source and receiver.

The mitigation can be done by inserting a soft element to reduce the stiffness $K_{R}$ of the receiver to $K_{R}{ }^{*}$. Thus the force transfer is reduced

$$
\left.\frac{F_{2}}{F}\right|_{\text {with }}=\frac{K_{R}{ }^{*}}{K_{Q}+K_{R}{ }^{*}} .
$$

The ratio $\mathrm{E}$ of the force transfer function with and without the mitigation measure is called the effectiveness of the mitigation measure

$$
E=\frac{\left.\frac{F_{2}}{F}\right|_{\text {without }}}{\left.\frac{F_{2}}{F}\right|_{\text {with }}}=\frac{K_{R}}{K_{Q}+K_{R}} \frac{K_{Q}+K_{R}^{*}}{K_{R}^{*}}=\frac{K_{R}}{K_{R}{ }^{*}} \frac{K_{Q}+K_{R}^{*}}{K_{Q}+K_{R}} .
$$

In special situations, namely for 1-dimensional systems, the effectiveness of a mitigation measure can be calculated by simpler formulas without specifying the excitation and the output quantity. If the mitigation measure is specified by the following stiffness relation

$$
\begin{aligned}
& F_{1}=K_{11} u_{1}+K_{12} u_{2} \\
& F_{2}=K_{21} u_{1}+K_{22} u_{2}
\end{aligned}
$$

where the index 1 holds for the source side and index 2 for the receiver side. These two equations and the two equations (21) can be solved to get the transfer function of the force

$$
\begin{aligned}
& F_{0}=\left(K_{11}+K_{Q}\right) u_{1}+K_{12} u_{2} \\
& 0=K_{21} u_{1}+\left(K_{22}+K_{R}\right) u_{2}
\end{aligned}
$$

which yields

$$
\begin{aligned}
& u_{1}=-\frac{K_{22}+K_{R}}{K_{21}} u_{2} \\
& F=\left(-\frac{\left(K_{11}+K_{Q}\right)\left(K_{22}+K_{R}\right)}{K_{21}}+K_{12}\right) u_{2}
\end{aligned}
$$


The ratio of the transfer functions without and with mitigation system yields the effectiveness or insertion loss

$$
E=\frac{\left.\frac{F_{2}}{F}\right|_{\text {without }}}{\left.\frac{F_{2}}{F}\right|_{\text {with }}}=\frac{\left(K_{11}+K_{Q}\right)\left(K_{22}+K_{R}\right)-K_{12} K_{21}}{-K_{21} K_{R}} \frac{K_{R}}{K_{Q}+K_{R}}=\frac{\left(K_{11}+K_{Q}\right)\left(K_{22}+K_{R}\right)-K_{12} K_{21}}{-K_{21}\left(K_{Q}+K_{R}\right)}
$$

The insertion loss is independent of the excitation load and the output quantity.

This formula can be specialised to the most simple mitigation measure. A single spring of stiffness $k_{I}$ has a stiffness matrix

$$
K=\left\lfloor\begin{array}{cc}
k_{I} & -k_{I} \\
-k_{I} & k_{I}
\end{array}\right\rfloor
$$

and therefore an effectiveness of

$$
E=\frac{\left(k_{I}+K_{Q}\right)\left(k_{I}+K_{R}\right)-k_{I}^{2}}{k_{I}\left(K_{Q}+K_{R}\right)}=\frac{\frac{1}{K_{Q}}+\frac{1}{K_{R}}+\frac{1}{k_{I}}}{\frac{1}{K_{Q}}+\frac{1}{K_{R}}}=1+\frac{1}{k_{I}} \frac{K_{Q} K_{R}}{K_{Q}+K_{R}}
$$

The insertion loss of a spring is the ratio of the total compliance of all elements to the compliance of source and receiver.

If the mitigation measure is described by a transfer matrix $\mathrm{T}$ as

$$
\begin{aligned}
& F_{1}=T_{11} F_{2}+T_{12} u_{2} \\
& u_{1}=T_{21} F_{2}+T_{22} u_{2}
\end{aligned}
$$

these two and the source and receiver equations (note the opposite sign convention of $F_{2}$ in the transfer matrix approach)

$$
\begin{aligned}
& K_{Q} u_{1}=-F_{1}+F \\
& K_{R} u_{2}=+F_{2}
\end{aligned}
$$

can be solved as

$$
\begin{aligned}
F-K_{Q} u_{1} & =T_{11} F_{2}+T_{12} u_{2}=T_{11} F_{2}+\frac{T_{12}}{K_{R}} F_{2} \\
u_{1} & =T_{21} F_{2}+T_{22} u_{2}=T_{21} F_{2}+\frac{T_{22}}{K_{R}} F_{2}
\end{aligned}
$$

and finally

$$
F=\left(T_{11}+\frac{T_{12}}{K_{R}}+K_{Q} T_{21}+\frac{K_{Q} T_{22}}{K_{R}}\right) F_{2} .
$$

The effectiveness E can be given as 


$$
E=\frac{\left.\frac{F_{2}}{F}\right|_{\text {without }}}{\left.\frac{F_{2}}{F}\right|_{\text {with }}}=\frac{K_{R} T_{11}+T_{12}+K_{R} K_{Q} T_{21}+K_{Q} T_{22}}{K_{Q}+K_{R}}
$$

As an example for a mitigation element described by a transfer matrix, a single mass is considered. A single mass $m$ has a transfer matrix

$$
T=\left\lfloor\begin{array}{cc}
1 & -m \omega^{2} \\
0 & 1
\end{array}\right\rfloor
$$

and the following effectiveness as a mitigation measure

$$
E=\frac{K_{R} T_{11}+T_{12}+K_{R} K_{Q} T_{21}+K_{Q} T_{22}}{K_{Q}+K_{R}}=\frac{K_{R}-m \omega^{2}+K_{Q}}{K_{Q}+K_{R}}=1-\frac{m \omega^{2}}{K_{Q}+K_{R}}
$$

For this case, the insertion loss is the ratio of the total dynamic stiffness of all elements to the dynamic stiffness of source and receiver. According to these formula, the mass must be dynamically stiff compared to the source and receiver, to yield a high effectiveness, whereas the spring $k_{I}$ in equation (27) must be soft compared to the source and the receiver.

\section{REFERENCES}

[1] L. Auersch, Dynamics of the railway track and the underlying soil: the boundaryelement solution, theoretical results and their experimental verification. Vehicle System Dynamics 43, 671-695, 2005.

[2] L. Auersch, Wave propagation in the elastic half-space due to an interior load and its application to ground vibration problems and buildings on pile foundations. Soil Dynamics and Earthquake Engineering 30, 925 - 936, 2010.

[3] L. Auersch, Dynamic axle loads on tracks with and without ballast mats - numerical results of three-dimensional vehicle-track-soil models. Journal of Rail and Rapid Transit 220, 169-183, 2006.

[4] L. Auersch, The dynamic behaviour of slab tracks on homogeneous and layered soils and the reduction of ground vibration by floating slab tracks. Journal of Engineering Mechanics 138, 923-933, 2012.

[5] L. Auersch, Simplified methods for wave propagation and soil-structure interaction: The dispersion of layered soil and the approximation of FEBEM results. C. Soize, G. Schuëller eds. Proc. $6^{\text {th }}$ Int. Conf. on Structural Dynamics (EURODYN 2005), Paris, France, 2005, 1303-1309

[6] L. Auersch, 2- and 3-dimensional methods for the assessment of ballast mats, ballast plates and other isolators of railway vibration. International Journal of Acoustic and Vibration 11, 167-176, 2006.

[7] R. Wettschureck, U. Kurze, Einfügungsdämmaß von Unterschottermatten. Acustica 58, 177-182, 1985. 
[8] DIN V45673-4, Mechanische Schwingungen - Elastische Elemente des Oberbaus von Schienenfahrwegen - Teil 4: Rechnerische Ermittlung der Einfügungsdämmung im eingebauten Zustand. Beuth Verlag, Berlin, 2008.

[9] L. Auersch, Zur Parametererregung des Rad-Schiene-Systems: Berechnung der Fahrzeug-Fahrweg-Untergrund-Dynamik und experimentelle Verifikation am Hochgeschwindigkeitszug Intercity Experimental. Ingenieur-Archiv 60, 141-156, 1990.

[10] L. Auersch, The excitation of ground vibration by rail traffic: Theory of vehicle-tracksoil interaction and measurements on high-speed lines. Journal of Sound and Vibration 284, 103-132, 2005. 